\title{
"Limbr, A Novel Personalized Mobile Application For Discogenic Chronic Low Back Pain: A Prospective Pilot Study"
}

Vijay B. Vad

Hospital for Special Surgery

Antonio Madrazo-lbarra ( $\nabla$ amadrazoi@live.com )

Hospital for Special Surgery

Deborah Estrin

Cornell Tech

Kaitlin M Carroll

Hospital for Special Surgery

Deneen Vojta

UnitedHealth Group (United States)

Amoli Vad

Hospital for Special Surgery

Camilla Trapness

Hospital for Special Surgery

John P. Pollack

Cornell Tech

\section{Research Article}

Keywords: Low back pain, Chronic low back pain, Exercise therapy, Telemedicine, Mobile applications, Self-management

Posted Date: March 8th, 2022

DOI: https://doi.org/10.21203/rs.3.rs-1108050/v1

License: (c) (i) This work is licensed under a Creative Commons Attribution 4.0 International License.

Read Full License 


\section{Abstract}

Background: Exercise has showed to reduce pain and improve function in patients with chronic lower back pain (CLBP), however, outcomes have been inconsistent. Limbr is a mobile application (app) developed to increase patient adherence to the exercise program, and enhance exercise's technique for better results.

Methods: Patients 18 to 65 years of age, with CLBP (more than 3 months) and evidence of lumbar disc pathology by magnetic resonance imaging (MRI) were enrolled. Patients' symptomatology was prospectively evaluated at baseline and during 3 months of using the Limbr app with patient-reported outcome measures (PROMs) including: the Visual Analog Scale for pian (VAS), the Oswestry Disability Index (ODI), and the Your Activities of Daily Living (YADL) score. Patients' compliance and satisfaction were evaluated at the end of the follow-up.

Results: Seventy-five patients with CLBP were enrolled in the study. All patients had a significant improvement from baseline to final follow-up in all PROMs. Average VAS scores decreased from $5.17 \pm$ 2.1 at baseline to $3.8 \pm 2.6$ at final follow-up $(P=0.016)$, and ODI scores from $25.7 \pm 13.8$ to $18.2 \pm 13.6(P=$ 0.001). Patients showed a significant decrease in the number of pain medications taken during a week $(P=0.001)$. Overall compliance with the app was greater than $52 \%$, with almost $65 \%$ of the patients rating the overall experience as good or excellent.

Conclusion: Limbr showed to be an effective tool in reducing low back pain symptomatology, as well as generating great compliance to an exercise program for patients with CLBP.

Trial registration number: 2/2/2017 NCT03040310 (ClinicalTrials.gov)

\section{Background}

It is estimated that 8 out of 10 people suffer from low back pain at some point in their life. [1] While most episodes are self-limited and resolve spontaneously, approximately $20 \%$ of low back pain episodes recur within 6 months of their initial occurrence. [2] The subset of patients with low back pain who continue to experience chronic symptoms is so large that chronic low back pain (CLBP) (defined as low back pain for $>3$ months) has been acknowledged as the most common cause of disability in individuals between 45 and 65 years old. [3, 4]Most of the time CLBP is non-specific meaning there is no evidence of structural abnormalities, however, discopathies are observed in $26-40 \%$ of patients, being this the second most common cause of CLBP. $[5,6]$ Societal and economic costs of CLBP are high, with most of these costs attributed to productivity loss due to incapacity. [7] Medical and surgical interventions may also be too expensive and catastrophic for patients suffering from CLBP. [8]

Important studies have demonstrated that exercise reduces pain and improves function in patients with CLBP. [9-11] Clinical implementation of exercise programs as a treatment for CLBP is challenging due to the lack of outcome consistency. Many of the differences in outcomes are due to the wide variety of 
exercise programs available, the use of supervised programs versus non-supervised programs, the low patient adherence to exercise programs, and the inconsistent recommendations of intensity and duration of programs. Patients suffering from CLBP report several barriers preventing them from following a regular exercise program going from the lack of motivation to the absence of self-involvement in the treatment. [12] A well-established affordable and accessible exercise program, that combines supervised with self-management interventions, and that focuses on strength/resistance and coordination/stabilization would be the perfect option to manage CLBP.

Mobile health (mHealth), referred to as the use of mobile phone applications for health-related measures, has emerged as an accessible tool for health care that offers psychological and medical assistance remotely reducing health care use, emergency room visits, and unnecessary expenses. Mobile apps related to health, promoting self-care and self-management, have greatly increased in the last couple of years. [13] Recently a study evaluating an app for chronic pain revealed mHealth is efficient for treating chronic pain, with better results observed in patients more engaged to the app. [14] There are currently multiple apps in the market trying to address CLBP, however many of them are low quality and lack evidence on their effectiveness. $[15,16]$

Limbr (pronounced "Limber") is the first mobile phone app developed to treat chronic lower back pain using the Back Rx rehabilitation program. The Back Rx program was developed in 2004 and has shown great success in preventing and treating CLBP. $[11,17]$ It consists of a 15 -minute exercise program that combines yoga and pilates exercises promoting strength, coordination, and stability. Limbr was released

to promote patient adherence to the program, encourage self-management, provide videos demonstrating the correct exercise technique, and offer professional coaching support and supervision. This pilot study is intended to evaluate the effectiveness of Limbr in treating low back pain in patients with discogenic CLBP and evaluate patient adherence to the Back Rx program, laying the basis for future randomized trials.

\section{Materials And Methods}

\section{Study Design}

This prospective pilot study was conducted at a single institution (NCT03040310, retrospectively registered on 2/2/2017 at ClinicalTrials.gov). The last patient evaluation was performed on September 2016. Patients were enrolled if they had all the following inclusion criteria: 18 to 65 years of age, chronic lower back pain ( $>3$ months of duration), evidence of lumbar intervertebral disc pathology on magnetic resonance imaging (MRI), computer literate, and owned a smartphone (iPhone models $5 \mathrm{~S}$ and later or Android models 2.3 or later). Exclusion criteria included: concurrent pathology that may contribute to the patient's axial low back symptoms (e.g., spondylolysis, spondylolisthesis, facet arthropathy), severe lumbar disc degeneration prior to beginning the Back Rx exercise program, history of lumbar spine surgery, or history of previous spine trauma. 
The study was performed according to Good Clinical Practice guidelines and principles of the Declaration of Helsinki. It was approved by the local ethics committee and the internal review board from the Hospital for Special Surgery (New York, NY). Written informed consent was obtained from all patients.

\section{Intervention}

Eligible patients were enrolled in a mHealth-based 3-month physical exercise program. Patients received a mobile phone app ("Limbr") free of charge to monitor and manage their CLBP. Prior to starting the 3month program, patients were given a detailed educational course on the app and all questions and concerns were addressed. The exercise program included self-directed rehabilitation video tutorials based on the Back Rx program, a virtual coach that reminded patients to perform their exercises, as well as motivated patients throughout the program; and periodic/sequential self-assessment patient-reported outcome measures to register pain and functionality.

\section{Evaluation}

The main objective of this study was to evaluate the effectiveness of Limbr in reducing lower back pain and improving functionality in patients with discogenic CLBP. Secondary outcomes were patient engagement to the app and compliance to the Back Rx program.

Symptomatology. Patient symptomatology was evaluated using patient-reported outcome measures (PROMs) including the Visual Analog Scale (VAS) for pain, Your Activities of Daily Living (YADL), the Oswestry Disability Index (ODI) for function, and the Medication for daily living. VAS, ODI, YADL were answered directly in the app by all patients at baseline; and at 3 weeks, 6 weeks, and 3 months after initiating the Back Rx program. The Medication for daily living was answered using the Limbr app daily at the end of each day.

YADL is an image-based survey that characterizes a patient's functional status using images representing activities of daily living from the Western Ontario and McMaster Universities Arthritis Index and Boston Activity Measure for Post-Acute Care. $[18,19]$ To complete the YADL, patients used the app and select images of activities during which they recently experienced lower back pain. Once an activity was selected, the patients graded the activity as "easy", "moderate", or "hard" depending on the difficulty patients had performing that activity. The difficulty was given a score of $0=$ easy, $1=$ moderate, and $2=$ hard. The full assessment included 47 images/activities and at the end of the assessment, all scores were added, divided by the total number of activities selected as pain triggers, and multiplied by 100 to obtain a percentage. The greater the percentage, the more symptomatic and less functional the patient was.

Medications of Daily Living is a visual app log used to evaluate "what" and "how many" medications the patient ingested in the last 24 hours. This evaluation was performed daily at the end of each day. A previous study explains both YADL and Medications of Daily Living in more detail. [20] 
Patient Compliance and Satisfaction. Patients were requested to watch and perform the exercises at least three times per week for them to be considered active. They confirmed they watched the whole video by answering a question that appeared at the end of each video. Participants were said to have completed the program (compliant) if they remained active for 12 weeks. A patient satisfaction questionnaire was performed at the end of the program (3 months).

\section{Statistical Analyses}

All continuous data with a normal distribution were expressed in terms of mean \pm SD; the categorical data were expressed as frequencies and percentages. Data with non-normal distribution were expressed in terms of median and variance. Shapiro-Wilk test was performed to assess the normality of continuous variables. All data with normal distribution were compared using Student t-test for continuous variables and chi-square for categorical variables. Nonparametric tests were used to compare data with nonnormal distribution. Pearson's correlation analysis was performed, $\mathrm{P}$ values $<0.05$ were considered statistically significant. Statistical Analysis was performed using SPSS software version 26 (IBM).

\section{Results}

A total of 75 patients with CLBP were enrolled in the study, 11 of which were lost to follow-up. Twenty-five patients used the app, but their pain resolved before finishing the 3-month program (6-10 weeks) and discontinued using the app. A total of 39 patients finished the 3-month program and were included in the final analysis. Patients who discontinued using the app due to symptom relief had statistically significantly lower body mass index (BMI) compared to patients who completed the follow-up (Table 1). All PROMs had a significant decrease from baseline to final follow-up (Fig. 1). Average VAS scores decreased from $5.17 \pm 2.1$ at baseline to $3.8 \pm 2.6$ at final follow-up $(P=0.016)$, ODI scores from $25.7 \pm$ 13.8 to $18.2 \pm 13.6(P=0.001)$, and YADL scores from $23.9 \pm 12.2$ to $18.1 \pm 12.2(P=0.009)$. Patients showed a decrease in the average pain medications taken each week since the first week of the program and reached statistical significance at final follow-up compared to baseline $(P=0.001)$ (Fig. 2). A weak positive correlation was obtained between YADL and ODI using full assessment YADL and baseline ODI scores $(R 2=0.2542, P=0.0062)$. Overall compliance with the app was greater than $52 \%(39 / 75)$, with almost $65 \%(25 / 39)$ of the patients rating the overall experience with this program as good or excellent. 


\section{Table 1. Demographic Characteristics of Patients}

\begin{tabular}{|lllc|}
\hline Characteristic & Finished Follow-up $(\mathbf{n = 3 9 )}$ & Discontinued App use $(\mathbf{n}=25)$ & P-value \\
\hline Sex, $\mathbf{n}(\%)$ & & $13(52)$ & \\
Female & $25(64)$ & $12(48)$ & 0.925 \\
Male & $14(36)$ & $45.0 \pm 14.7$ & 0.893 \\
Age, (years) & $44.4 \pm 15.6$ & $23.0 \pm 2.5$ & 0.024 \\
BMl kg/m² & $25.3 \pm 4.0$ & & \\
Data presented as mean \pm standard deviation unless otherwise specified. BMI = body mass index. \\
\hline
\end{tabular}

FIGURE 1. Patient-Reported Outcome Measures. Mean (95\% Cl); VAS = Visual Analog Scale, ODI = Oswestry Disability Index, YADL = Your Activities of Daily Living, PROM = Patient-Reported Outcome Measure. ${ }^{*}=P<0.05$

FIGURE 2. Average Pain Medications Taken in a Week. Mean (95\% Cl); 1 wk $=1$ week, Final $=3$ months. * $=$ $P<0.05$ Baseline versus Final

\section{Discussion}

This prospective pilot study evaluating the efficacy of Limbr demonstrates that this mHealth resource is effective in reducing pain and improving functionality in patients with discogenic CLBP, as well as an effective way to ensure an acceptable compliance to the Back Rx program. Patients' overall symptomatology had a statistically significant improvement from baseline to final follow-up (3 months) in all PROMs (VAS, YADL, ODI) (Fig. 1). According to the ODI score, known as the "gold standard" of low back functional outcome tools,[21] average scores at baseline indicated a "moderate disability", which improved to "minimal disability" after 3 months of using the Limbr app. Pain medication intake was decreased to more than half of the average medications taken weekly at baseline (Fig. 2). Overall compliance with the app was $52 \%$, suggesting a great engagement of patients with the exercise program and app. More than half of the patients (65\%) that finished the program affirmed to have a good or excellent overall experience.

Studies regarding symptom relief and functional improvement following an exercise program for CLBP have suggested exercise to be effective in reducing and preventing pain. [9, 10, 22-24] Amorim et al. [25] conducted a pilot randomized controlled trial evaluating the efficacy of the IMPACT app versus conventional lower back pain management in reducing pain and increasing functionality. They found the app to be more effective in function improvement, but equally effective in pain reduction than conventional treatment. Our study found Limbr to generate statistically significant pain and function improvements in patients with discogenic CLBP. This could be explained by the nature of the Limbr app which is based on the Back Rx program. This program combines both Yoga and Pilates exercises 
focusing on core strength, coordination, and stabilization. Searle et al. [10] showed that exercises focused on strength and resistance of the whole body and trunk, as well as coordination and stabilization are the most effective. The improvement in pain with the Back Rx exercises may be due to attenuating the proinflammatory processes in the body, increasing intervertebral disc nutrition and repair; and providing psychological benefits associated with chronic pain.

Home-based exercise programs encouraging self-management have demonstrated to decrease CLBP flares. [26] However, patient adherence to these home-based exercise programs has been reported to be around 30 to $50 \%$. [27, 28] Beinart et al. [29] in a systematic review revealed that the main reason for patients not adhering to self-managed exercise programs is lack of time to perform the exercises and the inability to remember them. This study evaluating the effectiveness of Limbr in increasing patient adherence to the Back Rx program showed Limbr to be an acceptable tool to reach up to $52 \%$ of engagement. This means more than half of the patients using the app performed the exercises at least 3 times a week. This adherence percentage obtained with Limbr is in the upper limit of what was previously described in the literature. The brief duration of the exercises performed daily in the Back Rx program (15 minutes) and the daily messages sent by the virtual coach motivating and reminding patients to do their exercises are two measures used by Limbr to increase patients' adherence to the program. A further study with a more in-depth analysis of patient adherence and what can be improved is suggested in the future.

Our study results show that Limbr is a great mHealth tool to deliver the Back Rx's program to patients with discogenic CLBP. This app showed to effectively decrease pain and increase function in patients suffering from an incapacitating and costly disease such as CLBP. Limbr is a cost-effective alternative for discogenic CLBP management which could help reduce catastrophic costs related to treatment and productivity losses due to incapacity. By decreasing pain, Limbr showed to be an efficient measure to decrease pain medication intake. This finding is crucial since we are living an important narcotic crisis, where almost $3 / 4$ of opiate-derived abusers started taking a legal prescription. [30] By decreasing the number of ingested medications, the risk of addiction and adverse effects related to medication also decreases.

One of the biggest limitations of this study is that there was no control group to compare the outcomes with. An adequate control group would have allowed a better understanding of the effectiveness of the Back Rx program in reducing symptoms, as well as the Limbr app in delivering the program. A second limitation is that $52 \%$ of the patients enrolled were analyzed at the final follow-up. This can be due to a selection issue explaining the 11 patients lost to follow-up or dropped out due to technical issues. However, the other 25 patients that did not finish the follow-up had a great outcome, with the pain resolving even before finishing the 3-month program; therefore they did not feel the need to continue. This is something expected with patients who are in a normal BMI since patients with greater BMI tend to respond slower to low back treatment. [31] This pilot study demonstrated that additional measures such as incentives could be used to increase patients' compliance in the real world. Another important limitation to mention is that this study was performed in a single institution and a single private practice which could have biased the results and decreased the response variability. A multicenter study is 
suggested to address the effectiveness of the app in a more pragmatic scenario, as well as a randomized placebo-controlled trial to evaluate the real effectiveness of the app.

\section{Conclusion}

In conclusion, Limbr showed to be effective in reducing low back pain symptomatology, as well as generating great compliance to an exercise program for patients with CLBP. Limbr will bring Back Rx to everyone's door, taking advantage of modern technology and promoting self-management.

\section{Abbreviations}

CLBP: Chronic low back pain; app: application; MRI: Magnetic Resonance Imaging; PROMs: PatientReported Outcome Measure; VAS: Visual Analog Scale; ODI: Oswestry Disability Index; YADL: Your Activities of Daily Living, mHealth: mobile health;

\section{Declarations}

\section{ACKNOWLEDGEMENTS.}

We would like to thank Deneen Vojta and The United Group for funding the study and trusting this amazing project that can have a great impact on reducing back pain, health care costs and opiate use.

\section{FUNDING}

The study was funded by the UnitedHealth Group. The funding body had no involvement in study design, data collection, analysis, interpretation of data, or writing the manuscript.

\section{AVAILABILITY OF DATA AND MATERIALS}

The datasets generated and/or analyzed during the current study are not publicly available due to patients' confidentiality but a coded copy of the dataset is available to all public upon request to the corresponding author.

\section{AUTHORS CONTRIBUTIONS}

VV: conducted, designed and supervised the study. AMI: assisted with data analysis, drafted, reviewed, and edited the manuscript; DE and JP: designed and conducted the study; KC: data analysis and assisted in the article draft, review and editing. DV: funded the study; AV and CT: assisted in data recollection.

\section{ETHICS APPROVAL AND CONSENT TO PARTICIPATE}

The study was approved by the local ethics committee and the internal review board from the Hospital for Special Surgery (New York, NY). Written informed consent was obtained from every patient prior to enrolling. 


\section{CONSENT FOR PUBLICATION}

Not applicable.

\section{COMPETING INTERESTS}

Author Vijay Vad owns the Back Rx trademark. All other authors have no conflicts of interest related to this research.

\section{References}

1. Balagué $F$, Mannion AF, Pellisé F, et al. Non-specific low back pain. Lancet. 2012;379(9814):482-491. doi:10.1016/S0140-6736(11)60610-7

2. Cassidy JD, Côté P, Carroll LJ, et al. Incidence and course of low back pain episodes in the general population. Spine. 2005;30(24):2817-2823.

3. Nicholas M, Vlaeyen JWS, Rief W, et al. The IASP classification of chronic pain for ICD-11: chronic primary pain. Pain. 2019;160(1):28-37. doi:10.1097/j.pain.0000000000001390

4. Frank JW, Kerr MS, Brooker AS, et al. Disability resulting from occupational low back pain. Part I: What do we know about primary prevention? A review of the scientific evidence on prevention before disability begins. Spine. 1996;21(24):2908-2917. doi:10.1097/00007632-199612150-00024

5. DePalma MJ, Ketchum JM, Saullo T. What is the source of chronic low back pain and does age play a role? Pain Med. 2011;12(2):224-233. doi:10.1111/j.1526-4637.2010.01045.x

6. Manchikanti L, Singh V, Pampati V, et al. Evaluation of the relative contributions of various structures in chronic low back pain. Pain Physician. 2001;4(4):308-316. doi:10.36076/ppj.2001/4/308

7. Vlaeyen JWS, Maher CG, Wiech K, et al. Low back pain. Nat Rev Dis Primers. 2018;4(1):52. doi:10.1038/s41572-018-0052-1

8. Schofield DJ, Callander EJ, Shrestha RN, et al. Back problems, comorbidities, and their association with wealth. Spine J. 2015;15(1):34-41. doi:10.1016/j.spinee.2014.06.018

9. Hayden JA, Ellis J, Ogilvie R, et al. Exercise therapy for chronic low back pain. Cochrane Database Syst Rev. 2021;9:CD009790. doi:10.1002/14651858.CD009790.pub2

10. Searle A, Spink M, Ho A, et al. Exercise interventions for the treatment of chronic low back pain: a systematic review and meta-analysis of randomised controlled trials. Clin Rehabil. 2015;29(12):1155-1167. doi:10.1177/0269215515570379

11. Vad VB, Bhat AL, Tarabichi Y. The role of the Back Rx exercise program in diskogenic low back pain: a prospective randomized trial. Arch Phys Med Rehabil. 2007;88(5):577-582. doi:10.1016/j.apmr.2007.02.008

12. Lim YZ, Chou L, Au RT, et al. People with low back pain want clear, consistent and personalised information on prognosis, treatment options and self-management strategies: a systematic review. J Physiother. 2019;65(3):124-135. doi:10.1016/j.jphys.2019.05.010 
13. Total mHealth market size worldwide 2025 forecast | Statista. Accessed October 28, 2021. https://www.statista.com/statistics/938544/mhealth-market-size-forecast-globally/

14. Ross EL, Jamison RN, Nicholls L, et al. Clinical integration of a smartphone app for patients with chronic pain: retrospective analysis of predictors of benefits and patient engagement between clinic visits. J Med Internet Res. 2020;22(4):e16939. doi:10.2196/16939

15. Machado GC, Pinheiro MB, Lee H, et al. Smartphone apps for the self-management of low back pain: A systematic review. Best Pract Res Clin Rheumatol. 2016;30(6):1098-1109. doi:10.1016/j.berh.2017.04.002

16. Nicholl BI, Sandal LF, Stochkendahl MJ, et al. Digital Support Interventions for the Self-Management of Low Back Pain: A Systematic Review. J Med Internet Res. 2017;19(5):e179. doi:10.2196/jmir.7290

17. Vad V, Occhiogrosso P. Back Rx. 2nd ed. Penguin Random House LLC; 2019.

18. McConnell S, Kolopack P, Davis AM. The Western Ontario and McMaster Universities Osteoarthritis Index (WOMAC): a review of its utility and measurement properties. Arthritis Rheum. 2001;45(5):453-461. doi:10.1002/1529-0131(200110)45:5<453::aid-art365>3.0.co;2-w

19. Jette DU, Stilphen M, Ranganathan VK, et al. Interrater Reliability of AM-PAC “6-Clicks" Basic Mobility and Daily Activity Short Forms. Phys Ther. 2015;95(5):758-766. doi:10.2522/ptj.20140174

20. Selter A, Tsangouri C, Ali SB, et al. An mHealth App for Self-Management of Chronic Lower Back Pain (Limbr): Pilot Study. JMIR Mhealth Uhealth. 2018;6(9):e179. doi:10.2196/mhealth.8256

21. Fairbank JC, Pynsent PB. The Oswestry Disability Index. Spine. 2000;25(22):2940-2952; discussion 2952. doi:10.1097/00007632-200011150-00017

22. Owen PJ, Miller CT, Mundell NL, et al. Which specific modes of exercise training are most effective for treating low back pain? Network meta-analysis. Br J Sports Med. 2020;54(21):1279-1287. doi:10.1136/bjsports-2019-100886

23. Zhu F, Zhang M, Wang D, et al. Yoga compared to non-exercise or physical therapy exercise on pain, disability, and quality of life for patients with chronic low back pain: A systematic review and metaanalysis of randomized controlled trials. PLoS ONE. 2020;15(9):e0238544. doi:10.1371/journal.pone.0238544

24. Matarán-Peñarrocha GA, Lara Palomo IC, Antequera Soler E, et al. Comparison of efficacy of a supervised versus non-supervised physical therapy exercise program on the pain, functionality and quality of life of patients with non-specific chronic low-back pain: a randomized controlled trial. Clin Rehabil. 2020;34(7):948-959. doi:10.1177/0269215520927076

25. Amorim AB, Pappas E, Simic M, et al. Integrating Mobile-health, health coaching, and physical activity to reduce the burden of chronic low back pain trial (IMPACT): a pilot randomised controlled trial. BMC Musculoskelet Disord. 2019;20(1):71. doi:10.1186/s12891-019-2454-y

26. Martínez de la Cal J, Fernández-Sánchez M, Matarán-Peñarrocha GA, et al. Physical Therapists' Opinion of E-Health Treatment of Chronic Low Back Pain. Int J Environ Res Public Health. 2021;18(4). doi:10.3390/ijerph18041889 
27. Du S, Hu L, Dong J, et al. Self-management program for chronic low back pain: A systematic review and meta-analysis. Patient Educ Couns. 2017;100(1):37-49. doi:10.1016/j.pec.2016.07.029

28. Saner J, Bergman EM, de Bie RA, et al. Low back pain patients' perspectives on long-term adherence to home-based exercise programmes in physiotherapy. Musculoskelet Sci Pract. 2018;38:77-82. doi:10.1016/j.msksp.2018.09.002

29. Beinart NA, Goodchild CE, Weinman JA, et al. Individual and intervention-related factors associated with adherence to home exercise in chronic low back pain: a systematic review. Spine J. 2013;13(12):1940-1950. doi:10.1016/j.spinee.2013.08.027

30. Cicero TJ, Ellis MS, Surratt HL, et al. The changing face of heroin use in the United States: a retrospective analysis of the past 50 years. JAMA Psychiatry. 2014 Jul 1;71(7):821-6. doi: 10.1001/jamapsychiatry.2014.366.

31. Wertli MM, Held U, Campello M, et al. Obesity is associated with more disability at presentation and after treatment in low back pain but not in neck pain: findings from the OIOC registry. BMC Musculoskelet Disord. 2016;17:140. doi:10.1186/s12891-016-0992-0

\section{Figures}

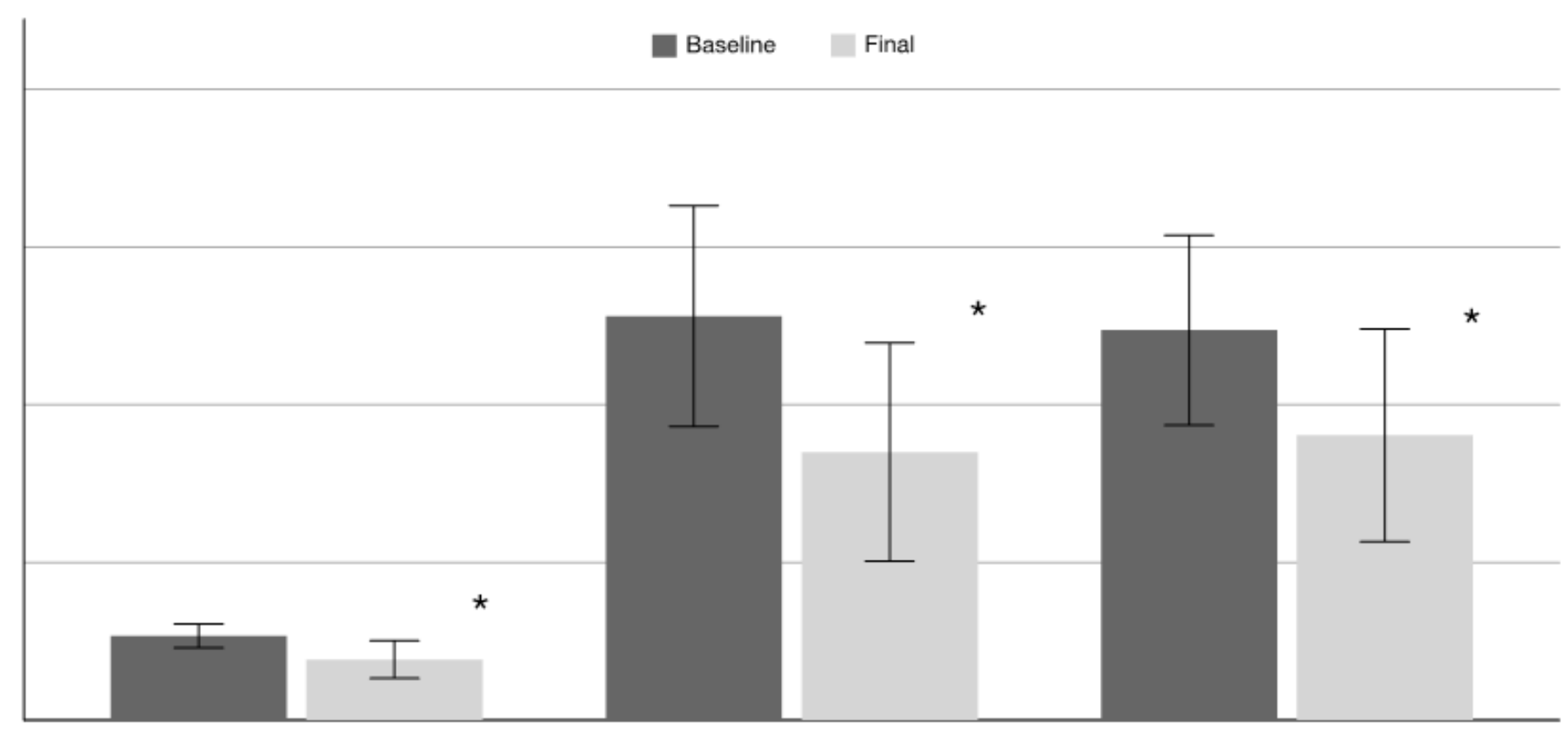

\section{Figure 1}

Patient-Reported Outcome Measures. Mean (95\% Cl); VAS= Visual Analog Scale, ODI= Oswestry Disability Index, YADL $=$ Your Activities of Daily Living, $\mathrm{PROM}=$ Patient-Reported Outcome Measure. ${ }^{*}=P<0.05$ 


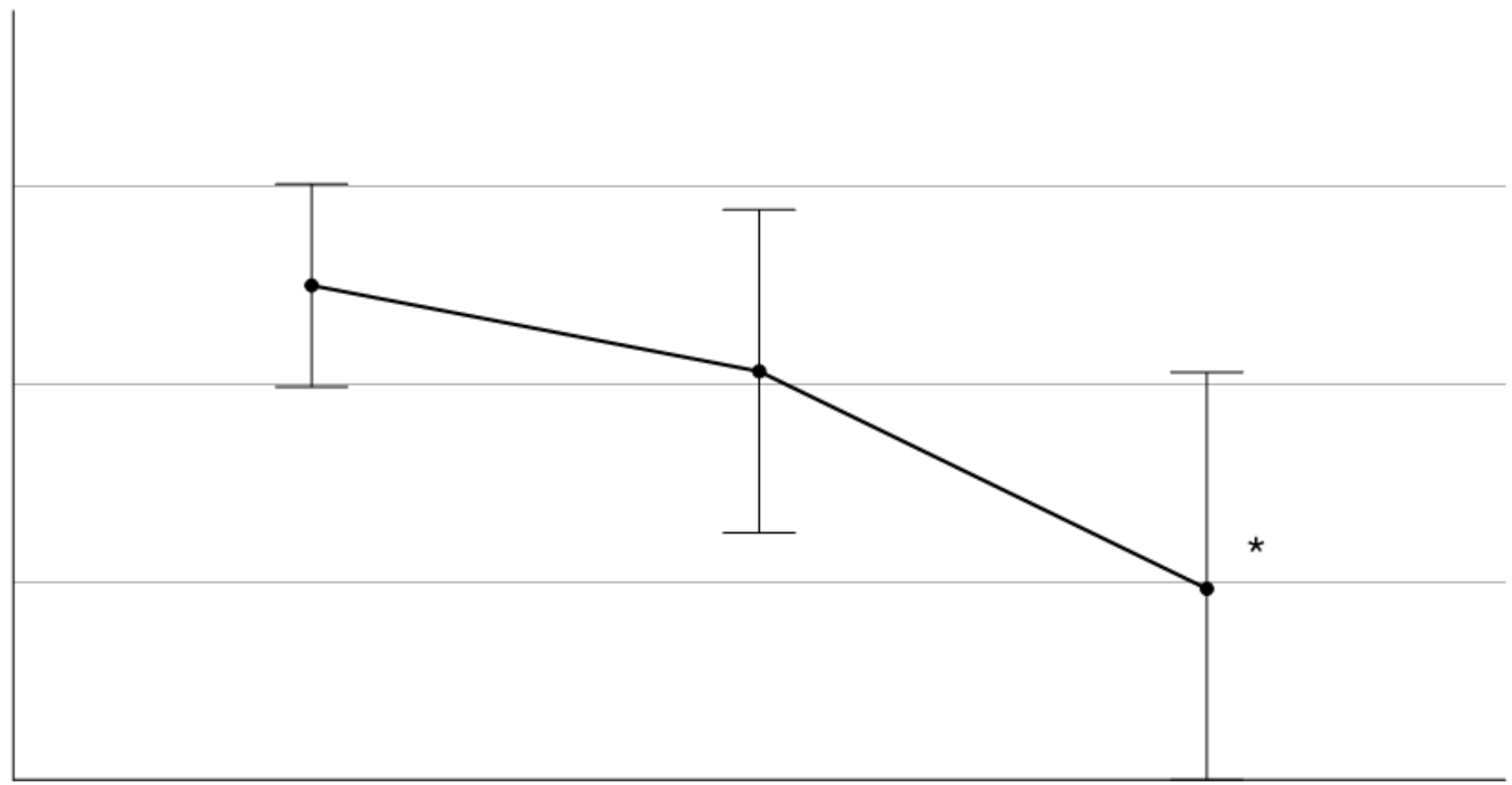

\section{Figure 2}

Average Pain Medications Taken in a Week. Mean (95\% Cl); 1 wk $=1$ week, Final $=3$ months. ${ }^{*}=P<0.05$ Baseline versus Final 